\title{
Pobreza y mortalidad perinatal en la población mapuche de la Araucanía
}

\author{
FLAVIO ROJAS, PH. D. ${ }^{1}$ \\ 1. Researcher, Odum Institute for Research in Social Science. University of North Carolina at Chapel Hill. \\ Odum Institute Staff, USA.
}

\begin{abstract}
Poverty and perinatal mortality among Mapuche Population in Araucania

Objectives: To establish a statistical relationship between poverty ad perinatal mortality at the individual level (death event) or contextual level (surrounding data). Patients and Method: Deaths of newborns in the 27 days of life were examined. The period between Jan 1st and Dec 31 of each year between 1996 and 2005 was considered. Individual data was verified by: gender, cause of death (ICD-10) and perinatal cause of death (P00-P96), ethnicity, age, district, community of procedence. Results: Poisson regressions showed statistical significance $\alpha<0.05$ for the relationship between poverty and perinatal mortality. This included smoothing for more stable estimation. All lineal general models showed statistical significance. Higher poverty level correlates with higher perinatal death. Chi Squared Scaled Pearson decreases a standard error in the interception estimator and the dependent variable (poverty). Conclusions: There is increased evidence that poverty and material risk have a clear effect upon population mortality, especially on vulnerable age groups. Ethnic variables show that poverty levels are significantly higher among Mapuche Population.

(Key words: Epidemiology, medical geography, models SIG/GIS, hierarchical methodology, spacial statistics, perinatal death).

Rev Chil Pediatr 2011; 82 (2): 93-104
\end{abstract}

\section{RESUMEN}

Objetivos: Establecer estadísticamente la relación entre pobreza y mortalidad perinatal tanto a nivel contextual (datos agregados) como a nivel individual (dato de muerte perinatal). Pacientes y Método: Se consideraron todas las muertes de recién nacidos en los primeros 27 días de vida entre el 1 de Enero y 31 de Diciembre de cada año entre 1996-2005. Los datos individuales se verificaron atributos por sexo, causa de muerte (ICD10) y causa de muerte perinatal (P00-P96), etnia, edad, comuna, unidad vecinal de procedencia. Resultados: Las regresiones efectuadas con datos de pacientes individuales ajustadas por proximidad espacial y contexto

Trabajo recibido el 21 de abril de 2010, devuelto para corregir el 09 de agosto de 2010, segunda versión el 21 de octubre de 2010, aceptado para publicación el 07 de febrero de 2011.

Correspondencia a:

Dr. Flavio Rojas

E-mail: frojas@email.unc.edu 
(modelos mixtos) con distribución Poisson, revelaron significación estadística $\alpha<0,05$ para la relación entre pobreza y mortalidad perinatal. Las regresiones con datos de pacientes individuales incluyeron técnicas de suavizado (smoothing) para obtener estimadores más estables. Los 5 ajustes con modelos generales lineales revelaron todos significación estadística. A mayor pobreza, mayor la probabilidad de muerte perinatal. Al ajustar las regresiones por sobre dispersión usando un Chi cuadrado con Pearson escalado, se advierte una disminución del error estándar tanto del estimador de intercepción como del estimador de la variable dependiente (pobreza). Conclusiones: Hay cada vez mayor evidencia que los niveles de pobreza y precariedad material tienen efectos discernibles en el riesgo de muerte de la población, especialmente en grupos étareos con mayores vulnerabilidades. Las variables étnicas han revelado ahora que los niveles de pobreza son significativamente mayores en la población mapuche.

(Palabras clave: Epidemiologías, Geografía Médica, Modelos SIG/GIS y métodos jerárquicos, estadística espacial, muerte perinatal).

Rev Chil Pediatr 2011; 82 (2): 93-104

\section{Introducción}

La presente investigación aborda por primera vez, la relación entre pobreza y mortalidad perinatal en la población de madres mapuches de la Araucanía en el período 19962005. Una década de observaciones y análisis se considera suficiente para determinar la existencia de eventos de naturaleza sistemática, profundamente enraizados en la estructura socio-económica regional de la Araucanía y que tienen consecuencias en el riesgo de vida y muerte perinatal.

El primer objetivo de este trabajo es la reconstitución de datos de mortalidad para todas las causas de muerte a partir de la información de los Registros de Defunción. A continuación se presenta un análisis y vinculación entre la información agregada de tasas de mortalidad por etnia y grupos etarios $(<5$ años $)$ con las áreas geográficas de procedencia de los pacientes con sus puntajes de pobreza verificados en la ficha CAS-2/familia. Finalmente, este trabajo aplica varios test estadísticos a nivel de pacientes individuales ajustado según su proximidad geográfica para verificar la significación espacial respecto de la relación entre pobreza y mortalidad.

\section{El espacio geográfico}

Desde que John Snow observara en 1855 las relaciones entre la distribución geográfica del cólera y las fuentes de agua contaminada que la población usaba para sus necesidades, quedó establecida la importancia de la epidemiología espacial como herramienta para las acciones en salud pública ${ }^{7}$. A estas intuitivas observaciones hay que agregar el elemento de distancia y el que observaciones que son más próximas entre si se comporten de modo distinto de aquellas que están más distantes, conlleva al concepto de cluster y por tanto, a datos que son dependientes el uno del otro. Este fenómeno se llama sobredispersión estadística de ahí que en el concepto de Riesgo Relativo considere la proximidad espacial como factor desconocido o riesgos no medibles de los factores de enfermedad y muerte.

\section{La pobreza mapuche identificada geográficamente}

Uno de los mayores avances tecnológicos y en la metodología estadística en los últimos años ha sido la posibilidad de incorporar las variables espaciales y representarlas en mapas geográficos con las epidemiologías de la población. La identificación de patrones espaciales permite conocer geográficamente la localización de bolsones de pobreza. La tecnología satelital de imágenes en desarrollo y su integración a mapas geo-referenciados están teniendo efectos insospechados en el conocimiento preciso de las demografías y distribución de las enfermedades, tendencias y dinámica de las 
poblaciones especiales, minorías y grupos de migrantes que habitan y se concentran en determinadas áreas de la ciudad y el espacio rural. La visualización de estos patrones mediante Sistemas de Información Geográfica (GIS) junto con la estimación y medición estadística de la distribución de eventos en el espacio con métodos frecuentistas y bayesianos ha traído un avance considerable en el diagnóstico y causas de las morbilidades y mortalidades de la población espacialmente identificada ${ }^{3,10,13-18}$.

Si los fenómenos de morbilidad y mortalidad están por tanto relacionados a factores de pobreza espacialmente identificables entonces deberemos relacionar los conceptos de Riesgo Relativo (RR) de fallecer en madres mapuches provenientes de áreas geográficas convenientemente desagregadas de modo de establecer los nexos correspondientes entre mortalidad y origen geográfico dentro de las Unidades Vecinales de la Región. La Encuesta CASEN aunque apropiada para establecer relaciones estadísticas entre atributos de la población, carece del nivel de desagregación espacial requerida para visualizar las relaciones entre etnia, grupos etáreos y sus niveles de pobreza con sus mortalidades asociadas.

La Ficha CAS-2/Familia contiene atributos socio-económicos de la población análogos a la Encuesta CASEN y similares a algunas categorías del Censo de Población y Vivienda de $2002^{6}$. El mapa geográfico siguiente proporciona la comparación de los puntajes de la Ficha CAS-2/Familia en 2005 y el Índice de Necesidades Básicas Insatisfechas (NBI) obtenidas de la base de datos del Censo de Población. Los valores de las NBI van desde 0 (cero), ausencia de necesidades básicas insatisfechas hasta 1, presencia máxima de NBI. Los puntajes de la Ficha CAS-2/Familia a su vez, van desde 442,34 puntos (máxima pobreza) a 612,10 puntos (mínima pobreza) (figura 1) (mapa 1). Según puede advertirse, a nivel sub-comunal, las Unidades Vecinales de la Ficha CAS-2/Familia $(\mathrm{N}=439)$ son bastante consistentes con los valores del índice NBI mostrados en los Distritos Censales $(\mathrm{N}=234)$ del Censo de Población. Hacia 2006, había 535000 personas inscritas en la Ficha CAS-2 de la Araucanía mientras que el Censo de Población contabili- zaba 834000 personas en la Región de la Araucanía. En consecuencia, alrededor del $64 \%$ de toda la población de la Novena Región estaba registrada en la Ficha CAS-2. ¿Como se distribuye entonces la población mapuche dentro de este espacio geográfico?. La figura 2 (mapa 2) desagrega longitudinalmente los datos CAS-2 para verificar la evolución de los puntajes y en última instancia comprobar si se observa una disminución de los mismos o eventualmente un cambio en la distribución de las áreas de pobreza regional a lo largo de los años.

Como puede advertirse, desde 1980 a 2005 la distribución de las áreas de pobreza regional se encuentra prácticamente inalterada durante el período considerado. Otro aspecto interesante son los puntos rojos que identifican la ubicación geográfica de la población mapuche. Presentan características de aglomeraciones ("clusters") mientras que la población nomapuche revela patrones aleatorios y dispersos por todo el territorio de la región. La población mapuche se concentra en las áreas geográficas de mayor pobreza regional, mientras que la población no mapuche se distribuye ubicua y heterogéneamente en el espacio geográfico. Esta distribución espacial de "cluster" para los asentamientos mapuches está verificada estadísticamente por test de Gettis-Ord y Moran (con probabilidad de 1\% que este patrón de cluster sea resultado de la casualidad).

La distribución conglomerada de la población (poblados) y reducciones mapuches se muestra en la figura 3 (mapa 3). Los mapas $3 b$ y $3 \mathrm{~d}$ muestran un patrón de distribución disperso y aleatorio (random). Los mapuches viven, territorialmente hablando, en las áreas geográficas de mayor pobreza e indigencia de la Región de la Araucanía. La figura 4 (mapa 4) muestra las reducciones mapuches, los poblados mapuches y no-mapuches y las tierras de la Red-Indígena, y las Unidades Vecinales con sus respectivos valores (SMR) suavizados. Las áreas suavizadas se intersectan bastante con las áreas de pobreza regional y desde luego con las áreas mapuches (reducciones, tierras asignadas y poblados). Las distribuciones conglomeradas, bolsones de pobreza. Podemos concluir que incluso introduciendo valores suavizados de las tasas estandarizadas de mortalidad perinatal, las 


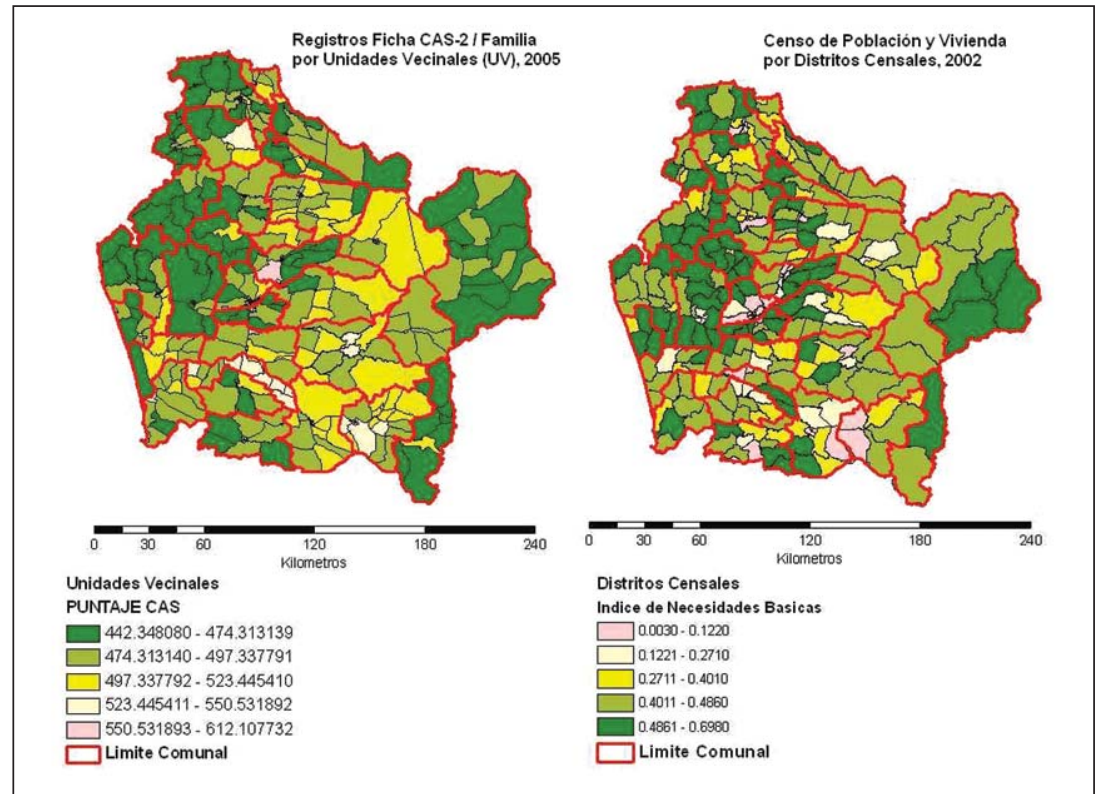

Figura 1. Mapa 1: Comparación mapa CAS-2/familia, unidades vecinales y valores puntajes CAS con mapa del Censo de población, distritos censales y valores del NBI.

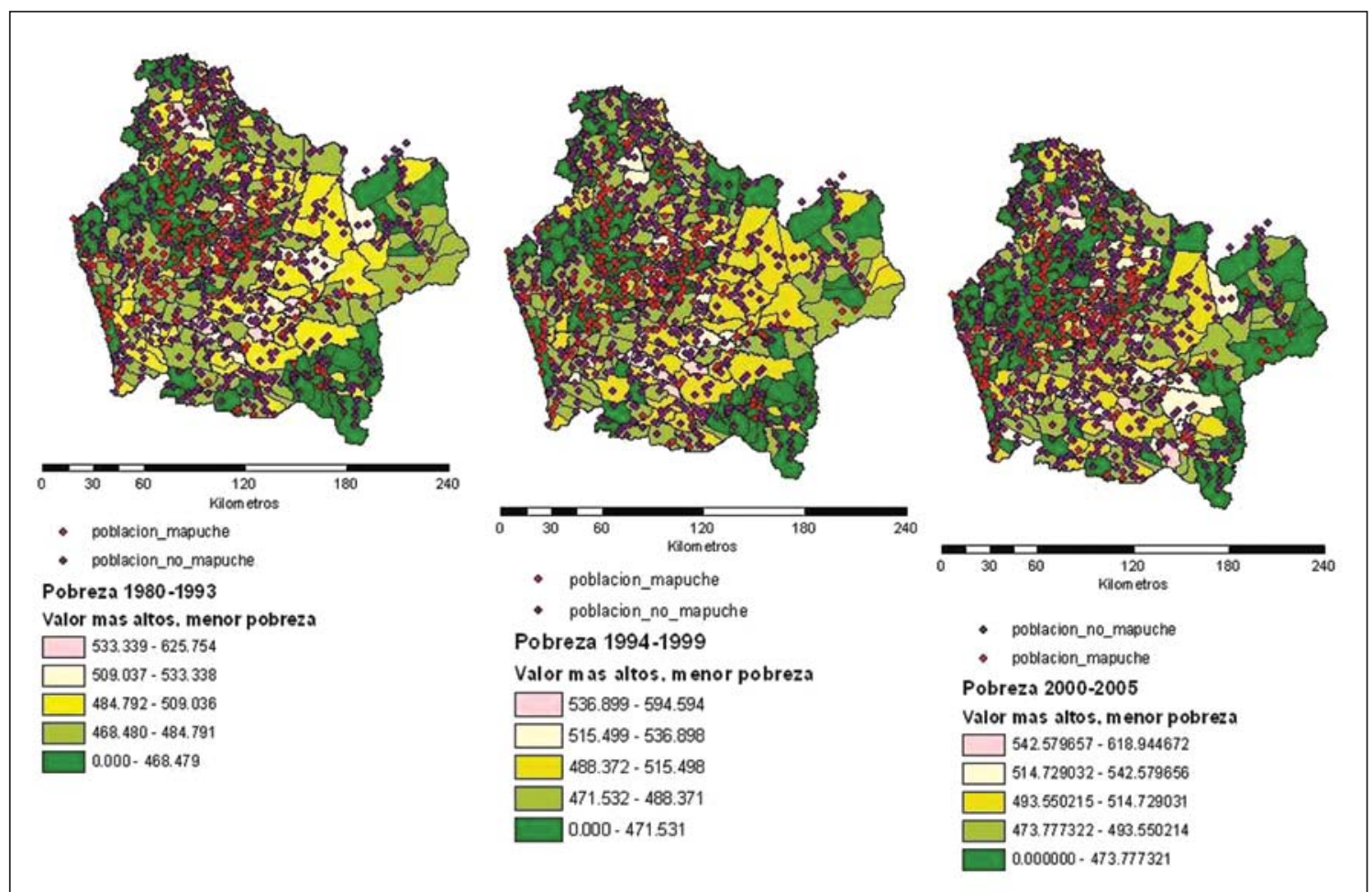

Figura 2. Mapa 2: Presentación longitudinal en tres tiempos, 1980-1992, 1994-1999, 2000-2005 de los puntajes Ficha CAS-2 según unidades vecinales y distribución geográfica censal de la población mapuche y no-mapuche de la Araucanía. 
áreas donde se ubican los valores más altos son precisamente las áreas geográficas de mayor pobreza y donde reside la población mapuche.

Los objetivos de este estudio fueron esta- blecer estadísticamente la relación entre pobreza y mortalidad perinatal tanto a nivel contextual (datos agregados) como a nivel individual (dato de muerte perinatal).
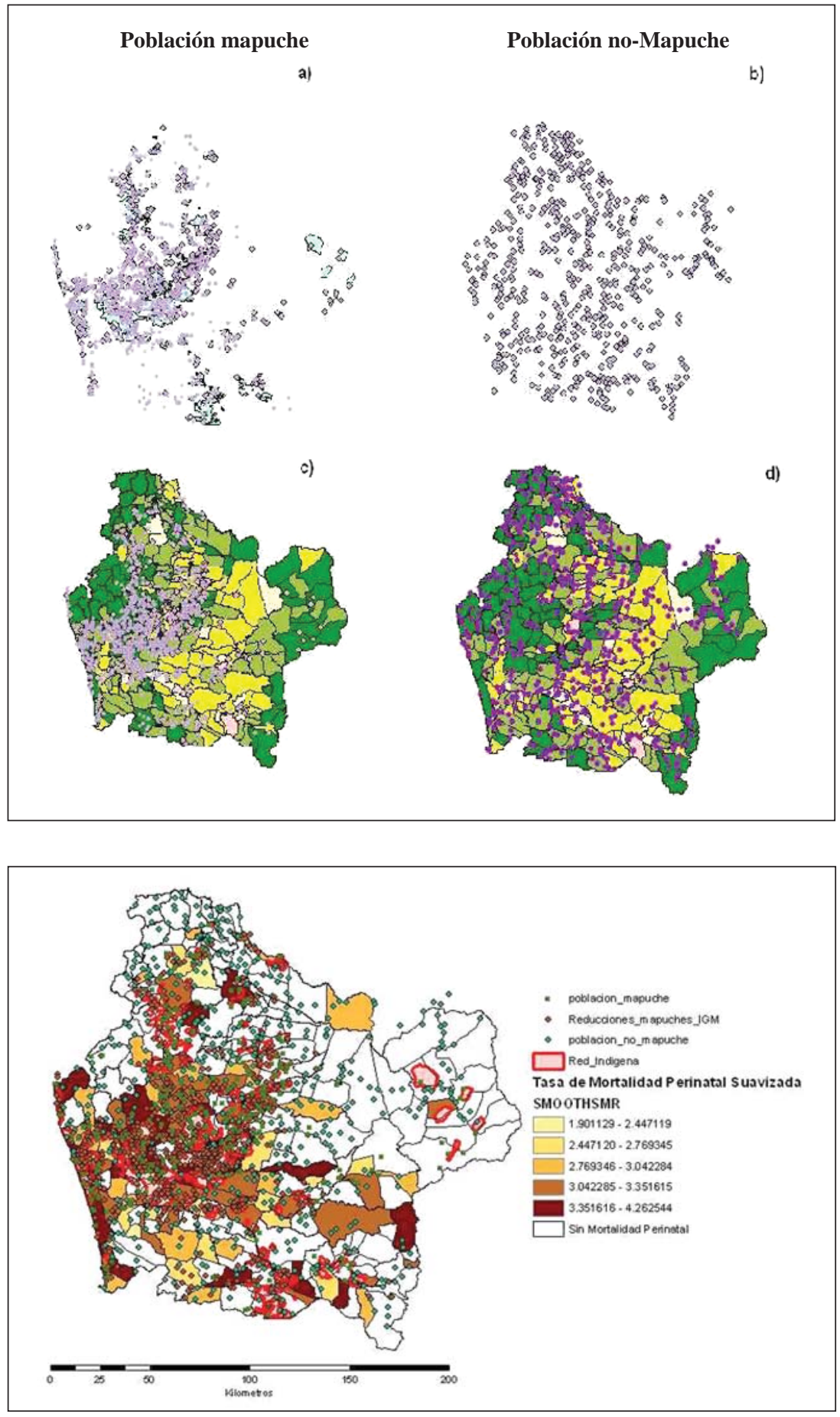

Figura 3. Mapa 3: Distribucion de la población en la Araucanía. Fuente: Instituto Geográfico Militar (IGM), Ficha CAS-2/Familia, Censo de Población.
Figura 4. Mapa 4: Tasas de mortalidad perinatal suavizada y distribución de la población mapuche y no-mapuche, Región de la Araucanía. 


\section{Pacientes y Método}

\section{Tipo de estudio}

Estudio longitudinal que incorpora simultáneamente variabilidad temporal con la heterogeneidad estructural ${ }^{1-4}$. Los datos provienen de los 32 hospitales de la Región de la Araucanía, 7 hospitales del Servicio Araucanía Norte, 15 hospitales del Servicio de Salud Araucanía Sur y 6 hospitales privados (hubo 4 hospitales que se fusionaron en el período).

\section{Pacientes}

Se ha usado en este estudio la información de los Registros de Defunción emitidos entre 1996-2005 por los Servicios de Salud Araucanía Norte y Sur entre el $1^{\circ}$ de Enero y 31 de Diciembre de cada año con diagnóstico clínico de "muerte perinatal" (ICD-10).

\section{Registro de información}

Se registró la comuna de procedencia, sexo, etnia, edad, RUT de la madre para todas las causas de muertes perinatales y códigos respectivos. Se consideraron las siguientes causales: feto y recién nacido afectado por factores maternos y por complicaciones en el embarazo, parto y nacimiento (P00-P04). Desórdenes relacionados con la duración de la gestación y crecimiento fetal (P05-P08). Trauma de nacimiento (P10-P15). Desórdenes respiratorios y cardiovasculares al sistema digestivo específicos al período perinatal (P20-P29). Infecciones específicas al período perinatal (P35-P39). Desórdenes hemorrágicos y hematológicos del feto y recién nacido (P50-P61). Desórdenes metabólicos y endocrinos transitorios específicos al feto y recién nacido (P70-P74). Desórdenes al sistema digestivo del feto y recién nacido (P75-P78). Condiciones que involucran el integumento y regulación de temperatura del feto y recién nacido (P80-P83). Otros desórdenes originados en el período perinatal (P90-P96).

Se generaron tasas estandarizadas directas e indirectas para todas las causas de muerte, tasas específicas y sus Coeficientes de Intervalo (CI) correspondiente, ajustadas por sexo, edad, etnia y comuna de procedencia. El RUT de la madre fue posteriormente empalmado con el
RUT proveniente de la base de datos de la Ficha CAS-2/Familia y sus correspondientes puntajes de pobreza. Se lograron identificar 654 casos de muerte perinatal entre 1996-2005, de los cuales 358 casos se pudieron establecer conexión domiciliaria de las madres con 171 de las 439 Unidades Vecinales de la Ficha CAS-2 distribuidas en las 32 Comunas de la Araucanía.

Desde el punto de vista metodológico la información anterior distingue aquellas causales de mortalidad que se establecen a nivel agregado: tasas especificas asociadas a variables étnicas de edad y sexo según año de ocurrencia, y aquellas que se establecen a nivel individual pero referidas causalmente a contextos de precariedad promedio de las Unidades Vecinales y los valores individuales de la Ficha CAS de las madres que registraron mortalidad perinatal entre los años 1996-2005 ${ }^{1}$.

Los Registros de Defunción guardados en planillas Excel fueron posteriormente exportados a un archivo $\mathrm{SAS}^{5}$ conjuntamente con los datos del Censo de Población y Vivienda ${ }^{6}$ donde se aplicó un programa para calcular tasas estandarizadas directas e indirectas y tasas no estandarizadas (proporciones binomiales simples) con sus respectivos intervalos de confianza exactos superiores e inferiores a nivel de comuna. Los datos fueron ajustados por edad, sexo y etnia obteniéndose las tasas de mortalidad especificas para cada una de las posibles combinaciones por edad, sexo, etnia, año y comuna, así como el correspondiente CI (95\%) para mapuches y no-mapuches, el Riesgo Relativo (RR) y CI correspondiente a ese RR.

Para los registros de pobreza se dispuso de la Ficha CAS-2/familia con la información individual de todas las personas que están inscritas desde 1980 hasta 2005 en las 31 comunas de la Araucanía (Chol-Chol paso a constituirse en comuna 32 como subdivisión de la comuna de Nueva Imperial, después del Censo). La Ficha CAS es una herramienta de focalización que identifica familias bajo la línea de la pobreza a partir de un puntaje de corte. Dependiendo el año, los puntajes CAS van desde 345 (máxima precariedad) hasta sobre los 750 puntos. Este puntaje final de corte es el resultado ponderado del ingreso, empleo, educación y calidad de la vivienda del grupo familiar. 
La presencia de sobredispersión en los datos de pobreza constatados por los test estadísticos (Gettis-Ord y Moran I) requiere del uso de regresiones espaciales que incorporen las variaciones extra-Poisson. En la práctica esto significa usar una estructura espacial residual para dar cuenta de mediciones que no han sido medidas pero que la variable confundida está espacialmente correlacionada. Una típica aproximación por ejemplo, es la de ajustar los efectos mixtos de un modelo de regresión para el conteo de enfermedades el cual incluye un término espacial residual para abordar la variabilidad que está espacialmente correlaciona$\mathrm{da}^{1}$. Hemos usado para este propósito el Macro de SAS, GLIMMIX y Proc GLIMMIX, Proc GLM y Proc GENMOD también de la familia SAS para procedimientos mixtos.

\section{Análisis estadístico}

Los datos del Registro de Defunción proveen estadísticas para establecer el nivel contextual con información agregada de tasas de mortalidad (todas las causas de muerte y causas especificas de muerte perinatal) ajustadas por sexo, edad $<5$ y etnicidad según comuna y datos correspondientes de RR comparados por sexo, etnicidad y edad sin referencia espacial. Los límites están basados en aproximación normal y distribución gamma, el límite superior fue calculado usando el método Fay-Feuer y el método Anderson-Rosenberg recomendado por $\mathrm{NCHS}^{8,9}$. Este método es supuestamente "conservador" ya que significa que es mayor que lo necesario para un límite superior y es mayor que el límite recomendado por NCHS. Los límites para el RR entre mapuches versus no-mapuches CI 95\% están basados en una distribución $\boldsymbol{F}$, ambas fueron aproximadas por distribuciones gamma y la razón de dos gammas es un $\boldsymbol{F}$.

El análisis estadístico a nivel de pacientes individuales se modela con una distribución Poisson donde hay dependencia espacial entre las unidades vecinales de donde provienen. Para la comparación espacial se utilizaron las tasas de mortalidad perinatal observadas (numerador) que se dividen con las tasas esperadas (denominador). Esta variable dependiente fue a su vez suavizada ("smoothed") para reducir inestabilidades derivadas de los contrastes entre áreas geográficas con poca población versus áreas con gran densidad, o contrastes por incidencias raras versus áreas con incidencias frecuentes de muerte perinatal. La idea básica es "pedir prestado" información de comunas vecinas para producir un mejor estimador asociado a cada Región ${ }^{10}$. Los puntajes CAS que tenían las pacientes se incluyeron en el modelo de regresión como variable independiente. El análisis fue hecho con el Macro Glimmix de SAS $9.2^{5}$ y el programa PROC GLIMMIX de SAS $9.2^{5}$ comparando 5 modelos de ajuste con significación estadística de un error tipo $\alpha$ de $5 \%(\mathrm{p} \leq 0,05)$.

\section{Resultados}

Las tasas de mortalidad comparadas de la población $<5$ años de la Araucanía, por sexo, grupos de edad y etnicidad 1996-2005, cada 2 años, se muestran en la tabla 1. Se observa que los niños(as) mapuches $<5$ años se mueren a tasas mayores que la población no-mapuche para ese mismo tramo de edad. Los Intervalos de Confianza (IC) no contienen el 0 (cero) y la tasa de mortalidad correspondiente cae dentro de los límites inferior-superiores del IC del parámetro poblacional ( $95 \%$ del IC). El Riesgo Relativo (RR) es $>1$ para la mortalidad infantil (todas las causas de muerte) de la población mapuche (IC 95\%, tabla 1). Ninguno de los valores en el rango superior-inferior contiene 0 (cero), demostrando que el riesgo relativo de fallecimiento para niños(as) mapuches es mayor que la de niños(as) no-mapuches $(\mathrm{p}<0,05)$.

Las principales causas de muerte de la población de la Araucanía, obtenidas por la vía de métodos directamente estandarizados por Organización Mundial de la Salud ${ }^{12}$ (WHO/ OMS), se muestran en la figura 5. Las tasas de muerte perinatal son recurrentemente a lo largo de los años superiores para la población mapuche comparado a la población no-mapuche de sexo masculino. Se verifican tendencias a la baja al compararse los últimos años con el año base 1996, sin embargo, las diferencias de Riesgo Relativo son recurrentemente mayores para los mapuches, y subsisten en el tiempo. 
ROJAS F.

Tabla 1. Cuadro comparado de todas las causas de muerte en la Araucanía por año, sexo, para grupo etario menor de 5 años y por etnia mapuche versus no-mapuche

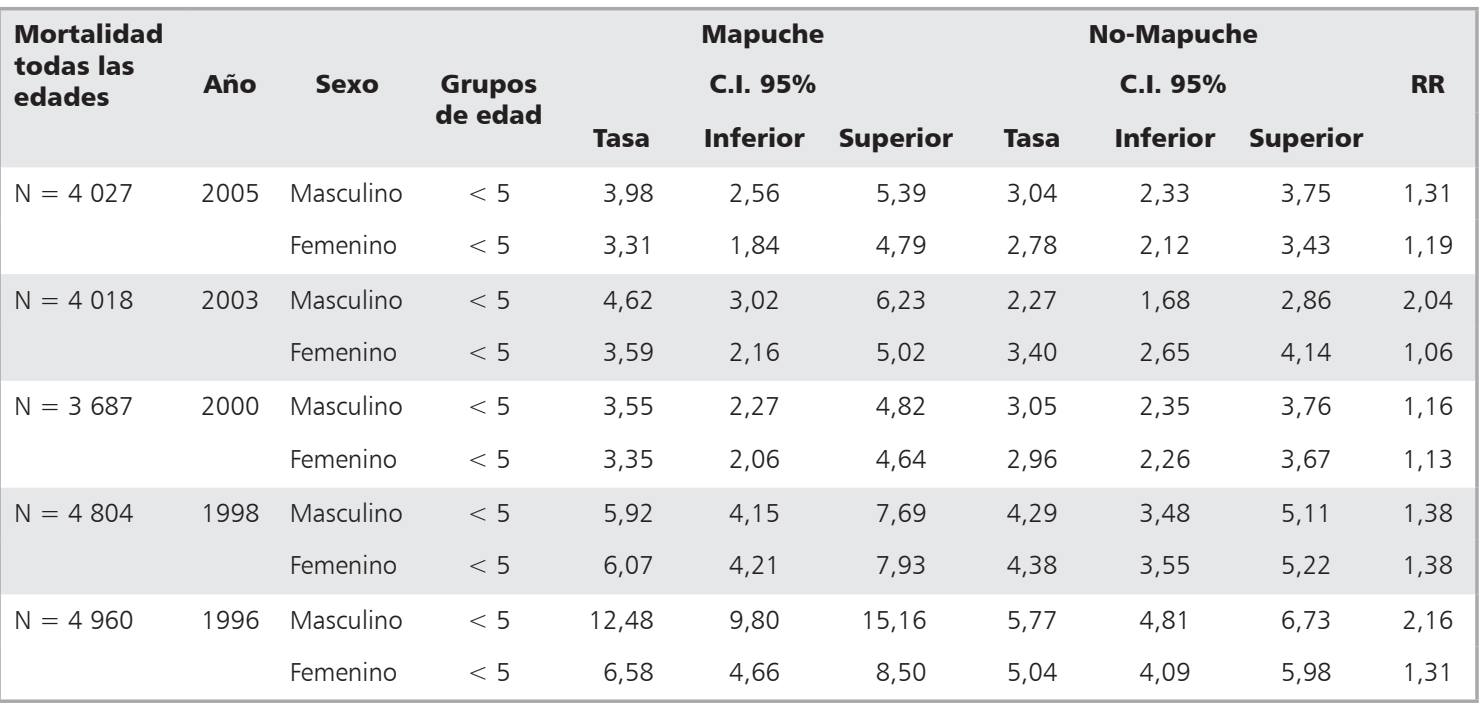

Fuente: Servicios de Salud Araucanía Norte y Sur, Registros de Defunción.

El Riesgo Relativo de mortalidad perinatal para tasas y edades específicas se muestra en la tabla 2. Las tasas de mortalidad perinatal son siempre mayores para las madres mapuches, exceptuando el año 2000 y 2003 para niñas fallecidas provenientes de madres no-mapuches. También las tasas de mortali- dad perinatal proveniente de madres mapuches muestra significación estadística (IC 95\%). El Riesgo Relativo de fallecer para niños provenientes de madres mapuches es siempre $>1$ y su IC $95 \%$ cae dentro del rango inferior-superior, ninguno de los cuales contiene al 0 .

Tabla 2. Cuadro comparado de las tasas de mortalidad por causas perinatales, región de la Araucanía para grupos de menos de 5 años, y por etnia mapuche versus no-mapuche

\begin{tabular}{|c|c|c|c|c|c|c|c|c|c|}
\hline \multirow[t]{3}{*}{ Mortalidad } & \multirow{3}{*}{ Año } & \multirow{3}{*}{ Sexo } & \multicolumn{3}{|c|}{ Mapuche } & \multicolumn{3}{|c|}{ No-mapuche } & \multirow{3}{*}{$\mathbf{R R}$} \\
\hline & & & & C.I. $95 \%$ & & & C.I. $95 \%$ & & \\
\hline & & & Tasa & Inferior & Superior & Tasa & Inferior & Superior & \\
\hline \multirow[t]{2}{*}{$N=116$} & 2005 & Masculino & 2,11 & 1,08 & 3,15 & 1,74 & 1,21 & 2,26 & 1,22 \\
\hline & & Femenino & 1,39 & 0,53 & 2,25 & 1,32 & 0,86 & 1,78 & 1,05 \\
\hline \multirow[t]{2}{*}{$N=104$} & 2003 & Masculino & 2,05 & 1,00 & 3,10 & 1,26 & 0,82 & 1,71 & 1,63 \\
\hline & & Femenino & 0,82 & 0,22 & 1,42 & 1,35 & 0,89 & 1,81 & 0,61 \\
\hline \multirow[t]{2}{*}{$N=107$} & 2000 & Masculino & 1,74 & 0,84 & 2,65 & 1,36 & 0,90 & 1,81 & 1,28 \\
\hline & & Femenino & 1,05 & 0,36 & 1,74 & 1,46 & 0,95 & 1,96 & 0,72 \\
\hline \multirow[t]{2}{*}{$N=143$} & 1998 & Masculino & 2,26 & 1,21 & 3,31 & 1,75 & 1,24 & 2,26 & 1,29 \\
\hline & & Femenino & 2,86 & 1,48 & 4,24 & 1,68 & 1,17 & 2,20 & 1,69 \\
\hline \multirow[t]{2}{*}{$N=184$} & 1996 & Masculino & 4,71 & 3,24 & 6,18 & 2,58 & 1,94 & 3,21 & 1,83 \\
\hline & & Femenino & 2,94 & 1,72 & 4,16 & 2,04 & 1,42 & 2,65 & 1,44 \\
\hline
\end{tabular}

Fuente: Servicios de Salud Araucania Norte y Sur, Registros de Defunción. 

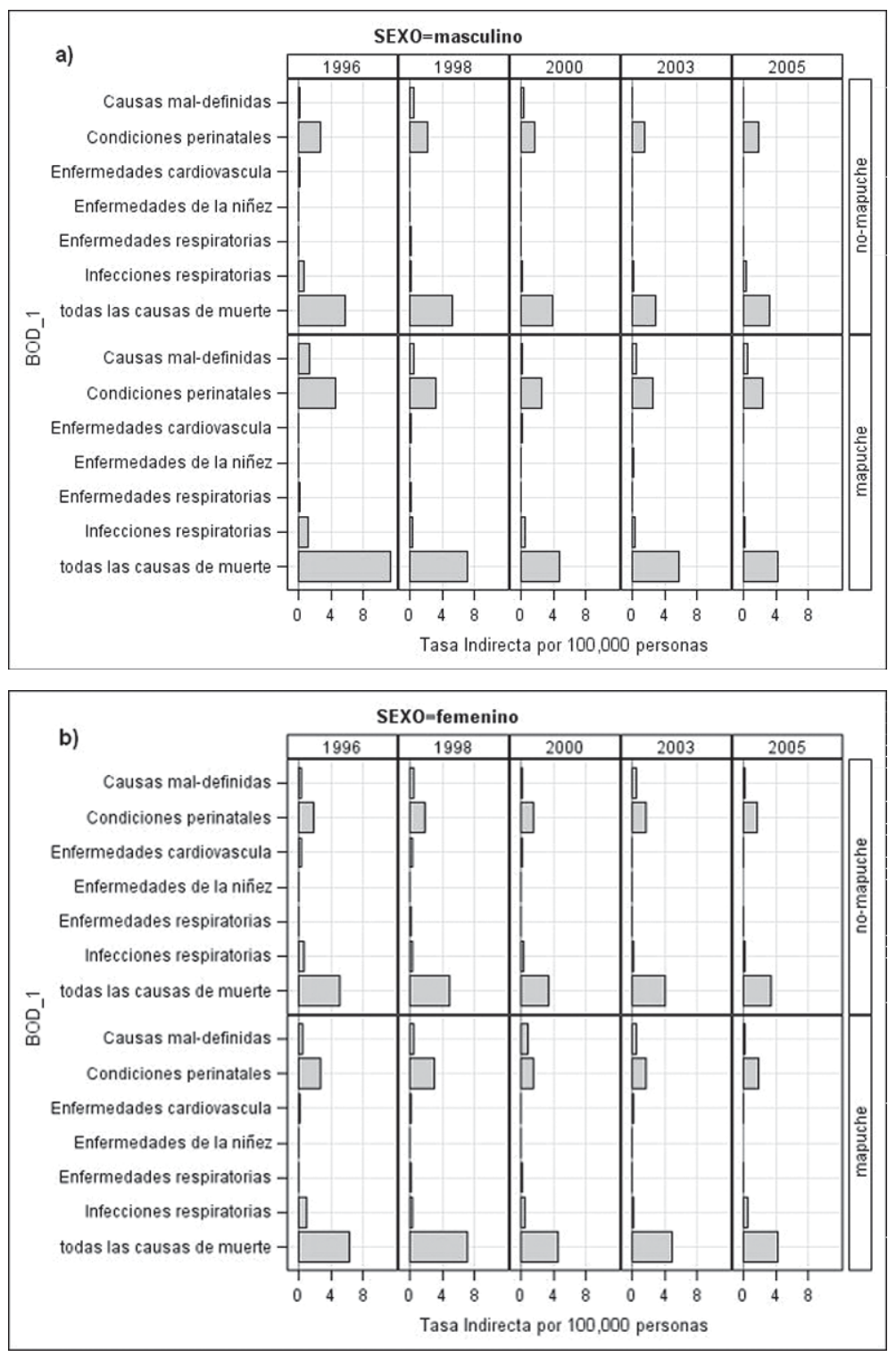

Figura 5. Gráfico 1a y 1b: Tasas de mortalidad directamente estandardizadas por cada 100.000 .

\section{Pobreza y mortalidad perinatal}

La tabla 3 presenta 5 ajustes hechos con 5 modelos generales lineales mixtos (GLMM) para correlacionar las muertes perinatales observadas, divididas por las muertes esperadas (SMR) (variable dependiente), y el puntaje CAS-2/Familia promedio que exhibía la Unidad Vecinal de donde provenían los 358 casos (variable independiente). Los métodos de aplicación para modelos generales lineares (GLM) y los modelos generales lineares mix- tos (GLMM) pueden encontrarse en Rojas ${ }^{19,20}$, y Rojas \& Suchindran ${ }^{21}$.

El modelo (1) de regresión Poisson sin ajuste por sobredispersión aunque significativo estadísticamente $(\mathrm{p}<, 0211)$ presenta un error estándar $(0,01224)$ mayor que los demás modelos (tabla 3$)$. El valor $(-0,0286)$ de la variable independiente $\hat{\beta}_{1}$ es negativo porque mientras menor el puntaje CAS-2 (mayor la pobreza), mayor es la probabilidad de muerte perinatal. El modelo (2) incluye ajustes por 
Tabla 3. Resultados de 5 ajustes con modelos generales lineales (glm) para tasas de mortalidad estandarizadas de mortalidad perinatal (variable dependiente) y pobreza (variable independiente)

\begin{tabular}{|c|c|c|c|c|c|c|}
\hline Modelos & $\hat{\beta}_{0}$ & $\hat{\beta}_{1}$ & $\hat{\sigma}_{s}^{2}$ & $\hat{\sigma}^{2}$ & $\hat{a}$ & p-value \\
\hline (1) PR & $-2,1229 \pm 0,6272$ & $-0,0286 \pm 0,0124$ & - & - & - & $<, 0211$ \\
\hline (2) $P R+O D$ & $-2,1223 \pm 0,3276$ & $-0,0286 \pm 0,0065$ & - & - & - & $<, 0001$ \\
\hline (3) Glimm (M) & $-2,1223 \pm 0,3276$ & $-0,0286 \pm 0,0065$ & - & - & - & $<, 0001$ \\
\hline (4) Glimm (P) & $-2,1228 \pm 0,3278$ & $-0,0285 \pm 0,0064$ & - & - & - & $<, 0001$ \\
\hline (5) Proc Glimm & $-2,1223 \pm 0,3276$ & $-0,0286 \pm 0,0064$ & - & - & - & $<, 0001$ \\
\hline
\end{tabular}

(1) PR = Proc Genmod, Regresión Poisson, sin ajuste por sobredispersión.

(2) $P R+O D=$ Proc Genmod, Regresión Poisson con Pearson Escalado para ajuste por sobredispersión.

(3) Glimm (M) = Macro Glimmix, Regresión Poisson sin ajuste por sobredispersión.

(4) Glimm (P) = Macro Glimmix, Regresión Poisson con efectos no espaciales aleatorios ajustados por sobredispersión.

(5) Proc Glimmix, Regresión Poisson sin ajuste espacial por correlación spacial.

sobre-dispersión usando un Pearson escalado (Scaled Pearson). Se advierte una disminución del error estándar tanto en la intercepción estimada $\hat{\beta}_{0}$ como en la variable independiente estimada $\hat{\beta}_{1}$ puntaje CAS-2. Los demás modelos generados por el GLIMMIX Macro como por PROC GLIMMIX de SAS (Statistical Analysis System), también revelan un buen ajuste y significación estadística $(\mathrm{p}<, 0001)$.

\section{Discusión}

Los hallazgos presentados en esta investigación revelan por primera vez la asociación directa entre la pobreza materna y mortalidad perinatal. Nunca antes se había establecido este vínculo quizás por la falta de información a nivel desagregado y de empalmar el micro dato territorial de modo de establecer simultáneamente las asociaciones ente el dato a nivel individual (atributos de la persona o paciente) y el contexto socio-económico de procedencia. En una investigación anterior (Rojas ${ }^{19}$ ) se había constatado la relación directa entre pobreza y enfermedades respiratorias agudas y mortalidad por esa misma causa para un año (2003). También la mayor incidencia de enfermedad y muerte (todas las causales de mortalidad) para la población mapuche en todas las edades se había logrado establecer con un diseño crossseccional válido para un solo momento en el tiempo. Esta investigación aporta una serie histórica de una década pero concentrando los análisis en la muerte perinatal. Esta causal de muerte da cuenta de modo concluyente que el riesgo de morir por causas perinatales es mayor para los recién nacidos mapuches y estas diferenciales de muerte y prevalencia para esta etnia se han mantenido inalteradas durante el período 1996-2005.

Los datos agregados de mortalidad perinatal y de riesgo relativo de muerte fueron también directamente asociados con la situación de pobreza de las madres de los fallecidos. Esto constituye un test definitivo para verificar la existencia de una relación estadísticamente significativa. Los 5 modelos de regresión indicaron una relación estadísticamente significativa: a mayor pobreza (menor los valores de puntaje CAS), mayor la probabilidad de muerte perinatal. Cabe hacer notar que la diversidad de los modelos permite distinguir entre aquellos que tienen ajuste espacial y los que no lo tienen. Esta distinción es importante porque los modelos sin ajuste espacial asumen independencia de los datos, suponen que las observaciones no tienen dependencia entre sí y que los errores se distribuyen aleatoriamente de un modo similar por todas las zonas del espacio geográfico. Este tipo de modelo tiende a subestimar los errores residuales y mostrar significación estadística aunque no exista. Este supuesto no se da en los datos usados en esta investigación. Diversos test usados (Moran I Global y Getis-Ord General) revelaron la dependencia espacial y autocorrelación de las observaciones de pobreza por lo que se de- 
bió usar un modelo mixto de regresión lineal (GLIMMIX) que corrige los factores de dispersión de los datos. Los estimadores de $\hat{\beta}_{0}$ y $\hat{\beta}_{1}$ son más o menos similares entre los modelos espaciales y los modelos sin ajuste espacial, sin embargo, los errores estándares asociados en los modelos espaciales son mucho menores que en los modelos no espaciales.

Las anteriores consideraciones y precondiciones para el logro de un buen modelo bioestadístico no deben dejar de lado la discusión sobre el enorme costo que tiene la pobreza para la sociedad en su conjunto en términos de la pérdida prematura de vidas sino la reflexión ética, legal y de política pública sobre cómo reducir o eliminar a) la pobreza circunscrita territorialmente a nivel de unidades vecinales (pobreza absoluta); b) reducir las disparidades entre los que muestran precariedad de los que no la tienen (pobreza relativa); c) reducir sobretodo las disparidades que tienen un trasfondo étnico (pobreza étnica) al comparar pacientes que tienen "uno o dos apellidos mapuches".

Estos hallazgos deberían alertar a médicos y especialistas en salud pública sobre como coordinar mejor la salud primaria municipalizada, en especial el control y seguimiento de los embarazos de la población carenciada, con las maternidades y salas de parto en los hospitales dependientes de los Servicios de Salud que son los destinatarios finales en la atención de los partos. Las experiencias de conectividad (intranet de salud) entre salud primaria y la columna vertebral hospitalaria dependiente del Ministerio de Salud podría facilitar una mayor coordinación entre una y otra instancia para reducir significativamente las esperas de atención de parto, cuidado neonatal y otros modos de intervención temprana que permitirían la prevención y reducción significativa de las muertes perinatales.

\section{Agradecimientos}

Ha contado con la generosa disponibilidad de medios para investigar a tiempo completo y valioso apoyo de mentoría y respaldo a participación en eventos internacionales en Europa y EE.UU. Sin el apoyo del Dr. Gary Koch, el Dr.
Chirayath M. Suchindran y colegas del Laboratorio de Consultoría en Biométrica (BCL) y sin la generosa participación de los Drs. Jaime Neira y Milton Moya, Director y Sub-director de los Servicios de Salud Araucanía Sur, y el Dr. Miguel Ángel Solar ex Director del Servicio de Salud Araucanía Sur, esta investigación no habría sido posible. Mi especial reconocimiento también va al Profesor Emérito en Bioestadística, Dana Quade,Ph.D. quien aportó en los programas SAS que se usaron para calcular las tasas de estandarización, riesgo relativo (RR) y coeficientes de intervalo usados en este trabajo.

\section{Referencias}

1.- Chen JT, Coull BA, Waterman PD, Schwartz J, Krieger $N$ : Methodologic implications of social inequalities for analyzing health disparities in large spatiotemporal data sets: An example using breast cancer incidence data (Northern and Southern California, 1988-2002. Statistics in Medicine 2008; 27: 3957-83.

2.- Lawson BA: Bayesian Disease Mapping: Hierarchical Modeling in Spatial Epidemiology 2008. Chapman \& Hall/CRC, Boca Raton, Florida.

3.- Banerjee S, Gelfand AE, Carlin BP: Hierarchical Modeling and Analysis for Spatial Data, Monographs on Statistics and Applied Probability, 2003. Chapman \& Hall/CRC, Boca Raton, Florida.

4.- Gelman A, Hill J: Data analysis using regression and multilevel/hierarchical models, 2007. Cambridge University Press, New York.

5.- $\quad$ SAS ${ }^{\circledR}$ Institute: SAS 9.2 Software, Carey, North Carolina.

6.- Instituto Nacional de Estadísticas, INE: XVII Censo Nacional de Población y VI de Vivienda, 2003. Empresa Periodística La Nación, Santiago de Chile.

7.- http://www.ph.ucla.edu/epi/snow/snowbook2.html

8.- Fay MP, Feuer EJ: Confidence intervals for directly standardized rates: a method based on the gamma distribution. Statistics in Medicine 1997; 16: 791-801.

9.- Anderson RN, Rosenberg HM: Age standardization of death rates: implementation of the year 2000 standard. National Vital Statistics Reports 1998, Vol 37, No. 3. Hyattsville, MD: National Center for Health Statistics.

10.- Waller LA, Gotway CA: Applied spatial statistics for public health data, 2004, John Wiley \& Sons, Hoboken, New Jersey. 
11.- CASEN: Encuesta de Caracterización Socioeconómica Nacional, Base de Datos, 2006, Ministerio de Planificación y Cooperación, Santiago de Chile.

12.- World Health Organization: Neonatal and Perinatal Mortality Country, Regional and Global Estimates (2006). Ver: http://whqlibdoc.who.int/publications/2006/9241563206_eng.pdf

13.- Bayari MJ, Berger JO: The Interplay of Bayesian and Frequentist Analysis. Statistical Science 2004; 19 (1): 58-80.

14.- Bland MJ: Bayesian and frequentists. British Medical Journal 1998; 317 (7166): 1151-60.

15.- Breslow N: Biostatistics and Bayes. Statistical Science 1990; 5 (3): 269-84.

16.- Gelman A, Hill J: Data analysis using regression and multilevel/hierarchical models, 2007, Cambridge University Press, New York.

17.- Coory MD, Willis RA, Barnett AG: Bayesian versus frequentist statistical inference for investigating a oneoff cancer cluster reported to a health department. BMC Medical Research Methodology 2009; 9: 30. http:// www.ncbi.nlm.nih.gov/pmc/articles/PMC2694210/

18.- Wakefield J, Stephens D: Bayesian Errors-in-Variables
Modeling in Dey Dipak K., Ghosh Sujit K. and Bani K. Mallick (Eds.) Generalized Linear Models A Bayesian Perspective, 2000, Marcel Dekker Inc., New York.

19.- Rojas F: Poverty determinants of acute respiratory infections among Mapuche Indigenous Peoples in Chile's Ninth Region of Araucanía, GIS and spatial statistics to identify the inequalities in health. International Journal of Health Geographics 2007; 6. http://www.ijhealthgeographics.com/content/6/1/26.

20.- Rojas F: En imprenta: Poverty Determinants of Acute Respiratory Infections in the Mapuche Population of Ninth Region of Araucanía, Chile (2000-2005): A Bayesian Approach with Time-space Modeling in McLafferty S. and Maantay J. (eds.), Geospatial Analysis of Environmental Health, Springer Series on "Geotechnologies and the Environment", 2010, New York, NY.

21.- Suchindran CM, Rojas F: Strategies for analyzing spatial-temporal demographic data in Population, Poverty and Health: Analytical Approaches, Edited by KK Singh, RC Yadav Arvind Pandey, Hindustan Publishing Corporation, India. viii, 384 p., tables, figs., ISBN 817075-088-1 (2009). http://www.vedamsbooks.com/ no59290.htm 